\title{
Some Exhibits at the Royal Society Conversazione
}

$\mathrm{T}$ HE first of this year's conversaziones at the Royal Society, held on May 9, produced as usual a number of interesting exhibits and demonstrations. Several have been the subject of recent communications in our correspondence columns, and descriptions of some of the remainder taken from the programme are printed below.

Mr. George H. Gabb showed a telescope of 1646 by Maria de Rheita, which is the earliest known dated optical instrument in the world. Maria de Rheita (1597-1660), a Capuchin Bohemian monk whose name was Antonius V. Schyrle before his monastic conversion, was the first to invent a terrestrial telescope with an image-erecting eyepiece of three lenses. He described its construction in a folio work, "Oculus Enoch atque Eliæ", published in 1645, a copy of which is in the library of the Royal Society. No example of his telescopes was hitherto known to have survived.

Mr. Henry Balfour showed an interesting collection of stone implements from Tasmania, from the fashioning of which the culture of the Tasmanians is regarded as corresponding to that of Cromagnon man in Europe.

Mr. R. W. Paul exhibited a simple apparatus for prolonged artificial respiration, which was designed at the suggestion of Sir William Bragg. It has already been used in one case of progressive muscular atrophy unceasingly for a period of eight months, and has prolonged the life of the patient, who, without aid, would die in less than two minutes. It comprises three main units : (1) an inflatable air bag, or belt, encircling the chest; (2) a pulsator which rhythmic. ally inflates the air baig; and (3) a controller governing the speed of the pulsator. The pulsator on its upward stroke inflates the air bag, causes a pressure to be applied to the chest and squeezes air out of the lungs. On the downward stroke of the pulsator, air is released from the bag, the chest resumes its normal position and air is inhaled. The volume of air inhaled can be varied by altering the pressure in the air bag. The apparatus is silent and automatic in operation, simple to adjust and easily transportable.

Dr. W. R. Jones showed microscope preparations illustrating his view that minerals other than uncombined silica can cause silicosis. Sections of many silicotic lungs show innumerable acicular fibres of sericite, a silicate of aluminium and potassium, which greatly outnumber the quartz particles. In the Kolar Goldfield, India, silicosis is rare, and sericite is also rare in the Kolar quartz; on the other hand, the gold-bearing rock worked on the Rand contains sericite, and many cases of silicosis occur.

The Entomological Department, Rothamsted Experimental Station (Dr. C. B. Williams and Mr. D. Morland) showed a light trap, which catches and kills the insects attracted to it at night and sorts them into eight groups according to the time of night at which they enter. The captures are correlated with various weather conditions including temperature, wind, humidity, the cloudiness of the sky and the duration of moonlight. A photoelectric method of measuring the cell space ratio in woods was demonstrated by the Forest Products Research Laboratory. The quantity of light transmitted by a suitably stained micro-section of the wood is measured as a percentage of the total quantity of light falling on the section. By adjusting the magnification of the projected image of the section, measurements may be made either on an integral number of annual rings, giving an average value of the cell space ratio, or on a small area confined to the spring or summerwood separately. Dr. F. G. Gregory and Mr. H. L. Pearse showed a self-recording apparatus for measuring changes in aperture of stomata. A glass cup attached to the lower side of a leaf by a gelatine washer is connected in series to a constant pressure aspirator through a variable capillary resistance, and air is thus drawn through the stomata. The pressure between the leaf and the resistance is recorded by a manometer, the varying level in which interrupts a beam of heat focused on a linear thermopile.

Some silica-glass from the Libyan Desert was shown by Dr. L. J. Spencer (Department of Mineralogy, British Museum (Natural History)). This material was recently discovered by $\mathrm{Mr}$. P. A. Clayton during the work of the Egyptian Desert Surveys in the unexplored region bordering on Italian Cyrenaica, about 500 miles south-west of Cairo. The material is a nearly pure silica-glass containing $\mathrm{SiO}_{2} 97.58$ per cent, pale greenish-yellow in colour and makes an effective gemstone. It is quite distinct from the fulgurites formed by lightning. It resembles most closely the still problematical tektites; the presence of a trace of nickel suggests a relation to the silica-glass found around meteorite craters.

Sir Gilbert Walker and Mr. A. Graham demonstrated the formation of artificial clouds. If a layer of liquid or air at rest is heated below or cooled above, the vertical instability will produce motion in polygonal cells ; but if the fluid moves with considerable shear, there will be longitudinal cells parallel to the direction of the shear. In air, a slow shear produced by sliding along the glass top of the containing trough produces transverse cells and an intermediate velocity a rectangular pattern. These can be identified with cloud forms. Prof. E. J. Baldes demonstrated micro-methods of measuring vapour pressure. It can be shown theoretically that the vapour pressure thermopile consisting of 50 couples of constantan silver, with wires of high thermal conductivity, is inefficient and that similar measurements can be made with a single thermocouple. The technique of measuring vapour pressures of small drops of liquid (1 mgm. or less) suspended from the junctions of a thermocouple was demonstrated. Mr. R. C. Brown showed some methods of studying capillary waves. Ripples produced on a liquid surface by a point- or line-source maintained by a valve oscillator are made to appear stationary by intermittent illumination of the same frequency. The frequency is determined by connecting an Osglim lamp across the oscillator output and allowing this to illuminate a stroboscopic disc controlled by a 50-cycle tuning fork. Thus measurements of wavelength and amplitude can be made over a range of frequency, the validity of Kelvin's equation tested, and surface tensions measured. It is also shown that a liquid surface over which ripples are passing may be used as a plane reflecting diffraction grating for visible light.

Prof. E. G. Coker and Prof. A. V. Hill combined 
to show some experiments on thermo-elasticity. The adiabatic thermal changes during extension and compression of materials have long been known, but measurement has been difficult. By employing a radiation thermopile and a galvanometer of high sensitivity and short period, the thermal effect of loading a specimen can be read on a scale, or recorded photographically, in a few seconds. The stresses in rigid materials can be measured with an accuracy comparable with any other known form of measurement, and strange to say, the order of the measurements depends on the coefficient of expansion of the material employed and not on Young's modulus and Poisson's ratio as in some other methods. Steel, brass, vulcanite and other bodies possessing a coefficient of expansion or contraction confirm this. In cases of plane complex stress, the measurements give the sum of the principal stresses. The adiabatic compression of various liquids, including water, has also been measured in this way, at pressures up to $1,500 \mathrm{lb}$. per sq. in., and the simple thermo-electrical arrangements used are such that the range of pressures can be extended almost indefinitely.

The Metropolitan-Vickers Electrical Company, Ltd., exhibited a portable noise-rneasuring apparatus, working on the aural balance principle. It consists essentially of a valve oscillator, a calibrated attenuator and a telephone earpiece. The oscillator generates current at 800 cycles which is fed to the telephone through adjustable attenuators. The apparatus is calibrated so as to indicate directly the intensity of the 800 cycle tone in the telephone in decibels above threshold of $0.0003 \mathrm{dynes} / \mathrm{sq}$. cm., measured in the ear or 0.00021 dynes/sq. cm., measured in free space. The observer places the telephone on one ear and presents the other to the complex noise to be measured. He then adjusts the attenuators until he judges the loudness of the 800 cycle note heard in the telephone to be equal to that of the complex noise. The equivalent value of the complex noise is then read in decibels above threshold from the settings of the attenuators.

A fractional seconds chronograph was shown by Mr. E. A. Nehan (Mathematical Department, Imperial College of Science). This instrument is designed to record visibly on paper tape, $0.01 \mathrm{sec}$. at $1 / 10$ th inch scale, up to four events simultaneously. A tuning fork controls a synchronous motor, which through a 3 -speed gear gives motion to a printing train and paper feed at the selected speed. Four inking pens record any event, translated as a simple make and break circuit, which mark on the tape is squared down to the time scale printed on the tape. The machine is portable, self-contained and worked from a 12-volt accumulator. Mr. J. Harvey, of the same Department, showed an integrator. By rolling a horizontal spur wheel on a rack in one direction and moving the rack perpendicularly, the axis of the wheel can be made to trace a curve whose Fourier coefficients are required. The wheel makes $n$ turns over a range of rack representing $0-2 \pi$. On a horizontal arm fixed to the axle of this wheel, is a wheel which rolls on the paper and registers the coefficients $a_{n}$ and $b_{n}$. Six harmonics can be found. The mechanism is adapted to find area, and first and second moments of area about an axis, on the principle of Amsler's moment integrator.

The National Physical Laboratory (Mr. R. A. Watson Watt, Mr. J. F. Herd and Mr. L. H. Bain. bridge-Bell) showed a cathode ray tube which has been made to serve as a magnetic compass. The cathode ray beam is deflected by the earth's magnetic field and may therefore be used as a compass needle free from inertia. In the present instrument light signals controlled by collector electrodes in the tube indicate any change in the magnetic bearing of the platform carrying the tube. Changes of a small fraction of a degree can be indicated. Among the exhibits by the Director of Scientific Research, Admiralty, was an apparatus which enables the deaf to hear by bone conduction. Amplified currents from a microphone are supplied to a coil wound direct on to a straight nickel iron wire which has a high magnetostriction coefficient. The wire vibrates with the frequency of the current and the vibrations may be transmitted to the cochlea through the bones of the head or jaw either by clenching the wire in the teeth or by pressing it on the mastoid bone behind the ear. The same Department also showed an automatic radio direction finder. This instrument is of the Bellini-Tosi type comprising two crossed frame coils and a goniometer, the search coil of which is rotated by an electric motor. The goniometer is connected to a radio receiver followed by a special rectifier and relay system which reverses the direction of the electric motor whenever the high frequency current in the search coil increases; thus, since the direction of rotation of the search coil is reversed whenever the minimum is passed, the search coil hunts a position at minimum.

\section{Interpretation of Evidence for the Recession of Nebulæ}

A

T Oxford on Tuesday, May 8, Dr. Edwin Hubble, of the Mount Wilson Observatory, delivered the Halley Lecture on "Red-Shifts in the Spectra of Nebulæ". As is indicated by the title, the lecture was concerned with a non-speculative account of those investigations, largely due to Dr. Hubble himself, which have led to the far-reaching speculations of de Sitter, Eddington, Milne, and others on the expansion of the universe. After a masterly account of the use of Cepheid variables in determining the distances of the nearer extra-galactic nebulæ, of the statistical methods involving the intrinsic luminosity of the average nebula for the distances of the remoter objects, and of the radial velocities or red-shifts measured by Slipher and Humason, Dr. Hubble developed in full the velocity-distance relation which bears his name. For the 150 nebulæ of which spectra have now been obtained, and which lie within a sphere of 150 million light years radius, he was able to show that the red-shifts, expressed as velocities, increase linearly by roughly one hundred miles per second for each million light years of distance; further, he was able to show that the difference between the linear relation for isolated nebulæ and for clusters of nebulæ is wholly the effect of selection, and thus to harmonise the results from all observed nebulæ.

The lecture concluded with an attempt to interpret the observed red-shift on the basis of the available observational material alone. If the red-shift is produced by some unspecified cause, Doppler effect being thus expressly excluded, then the brightness 